DESY 03-081

July 2003

\title{
Solving Recurrence Relations for Multi-Loop Feynman Integrals
}

\author{
Vladimir A. Smirnov ${ }^{a}$ and Matthias Steinhauser ${ }^{b}$ \\ ${ }^{a}$ Skobeltsyn Institute of Nuclear Physics, Moscow State University, \\ 119992 Moscow, Russia \\ ${ }^{b}$ II. Institut für Theoretische Physik, Universität Hamburg, \\ Luruper Chaussee 149, 22761 Hamburg, Germany
}

\begin{abstract}
We study the problem of solving integration-by-parts recurrence relations for a given class of Feynman integrals which is characterized by an arbitrary polynomial in the numerator and arbitrary integer powers of propagators, i.e., the problem of expressing any Feynman integral from this class as a linear combination of master integrals. We show how the parametric representation invented by Baikov [1] can be used to characterize the master integrals and to construct an algorithm for evaluating the corresponding coefficient functions. To illustrate this procedure we use simple one-loop examples as well as the class of diagrams appearing in the calculation of the two-loop heavy quark potential.
\end{abstract}

PACS numbers: 02.70.-c, 12.38.-t, 14.65.-q 


\section{Introduction}

In the recent years the art of evaluating multi-loop Feynman integrals has been driven to an impressive high level and very sophisticated methods and tools have been developed in order to cope with the enormous complexity one encounters at higher orders (see, e.g., the recent reviews [2]). A standard practical problem is the evaluation of a class of Feynman integrals corresponding to a given graph. The integrals differ by the integer powers of the propagators and the polynomials in numerators. Most methods heavily rely on the use of recurrence relations derived with the help of integration-by-parts (IBP) 3] identities. The recurrence relations are used to express a complicated integral in terms of simpler ones and, after repeated use, finally in terms of a small set of integrals, so-called master integrals, which can not further be reduced. Only for the latter a complicated integration is possibly necessary.

Any dimensionally regularized Feynman integral corresponding to a given graph, with the space-time dimension $d=4-2 \epsilon$ as a regularization parameter [4, can be represented in the form

$$
F(\underline{n})=\int \cdots \int \frac{\mathrm{d}^{d} k_{1} \ldots \mathrm{d}^{d} k_{h}}{D_{1}^{n_{1}} \ldots D_{N}^{n_{N}}},
$$

where $k_{i}, i=1, \ldots, h$, are loop momenta, $n_{i}$ are integer indices, underlined letters denote multi-indices, i.e., $\underline{n}=\left(n_{1}, \ldots, n_{N}\right)$, and the denominators are given by

$$
D_{a}=\sum_{i \geq j \geq 1} A_{a}^{i j} p_{i} \cdot p_{j}-m_{a}^{2},
$$

with $a=1, \ldots, N$. Irreducible numerators which cannot be linearly expressed through the given set of the denominators $D_{a}$ of the propagators are naturally treated as extra denominators raised to a negative power. The momenta $p_{i}$ are either the loop momenta $p_{i}=k_{i}, i=1, \ldots, h$, or external momenta $p_{h+1}, \ldots$ of the graph so that the denominators can be either quadratic or linear ${ }^{1}$ in the loop momenta $k_{i}$.

The IBP identities are obtained by applying the operator $\left(\partial / \partial p_{i}\right) \cdot p_{j}(i=1, \ldots, h$ and $j=1, \ldots, h, \ldots)$ to the integrand in Eq. (11). Due to the properties of dimensional regularization the resulting integral is zero which can symbolically be written as

$$
P_{i j}\left(I^{+}, I^{-}\right) F(\underline{n})=0 .
$$

$P_{i j}$ are polynomials in the operators that increase and lower indices, $\mathbf{I}_{a}^{+}$and $\mathbf{I}_{a}^{-}$, where

$$
\begin{aligned}
& \mathbf{I}_{a}^{+} F\left(\ldots, n_{a}, \ldots\right)=F\left(\ldots, n_{a}+1, \ldots\right), \\
& \mathbf{I}_{a}^{-} F\left(\ldots, n_{a}, \ldots\right)=F\left(\ldots, n_{a}-1, \ldots\right) .
\end{aligned}
$$

The basic idea is to use the relations of Eq. (3) in order to express any integral with a given value of the multi-index $\underline{n}$ in terms of simpler integrals corresponding to some

\footnotetext{
${ }^{1}$ Linear denominators usually appear in asymptotic expansions of Minkowski space Feynman integrals within the strategy of expansion by regions [5].
} 
finite family of multi-indices $\underline{n}_{i}$, where, usually, $\underline{n}_{i}=\left(n_{i 1}, \ldots, n_{i N}\right) \operatorname{consists}^{2}$ of $n_{i a}=0$, or 1 , or a negative integer value. Having in mind the experience collected in the process of solving recurrence relations for various classes of Feynman integrals, one may hope that, for any given class of Feynman integrals, the recursion problem can be solved, i.e., any Feynman integral $F(\underline{n})$, with given values of its indices, can be represented as a finite linear combination

$$
F(\underline{n})=\sum_{i \geq 1} c_{i}(\underline{n}) F\left(\underline{n}_{i}\right)
$$

with the normalization condition

$$
c_{i}\left(\underline{n}_{j}\right)=\delta_{i j} .
$$

The integrals $F\left(\underline{n}_{i}\right)$ are called master, basic or irreducible integrals. Our experience tells us that the coefficient functions $c_{i}(\underline{n})$ turn out to be rational functions of the dimension $d$, the masses and the kinematical invariants build from the external momenta, so that the non-trivial analytical dependence of the Feynman integrals on the dimensionful quantities and $d$ is completely contained in the master integrals.

To solve the problem of the reduction it is necessary to identify the master integrals and to construct an algorithm which allows to calculate the coefficients $c_{i}(\underline{n})$. In concrete situations, the realization of the reduction procedure to a set of master integrals turns out to be far from straightforward. Examples of recent attempts to construct systematic procedures for solving IBP recurrence relations can be found in [6, 17, 8, 9].

An alternative approach to solve recurrence relations in a systematic way was developed in [1]. This approach is based on an appropriate integral representation for the coefficient functions $c_{i}(\underline{n})$. In the case of vacuum Feynman integrals, this representation takes the form 1

$$
c_{i}(\underline{n}) \sim \int \ldots \int \frac{\mathrm{d} x_{1} \ldots \mathrm{d} x_{N}}{x_{1}^{n_{1}} \ldots x_{N}^{n_{N}}}[P(\underline{x})]^{(d-h-1) / 2},
$$

where the parametric integrals have to fulfill the essential condition that IBP can be applied. As one can see in the examples discussed in Section [3, the integrals in Eq. (7) can be either performed as closed contour integrals in the complex plane, or as iterated integrations over real one-dimensional domains. The latter is typically between roots of a quadratic polynomial. Furthermore, if the singularity of the $x_{i}$-integration is given by the factor $x_{i}^{-n_{i}}\left(n_{i}=1,2, \ldots\right)$ it is unreasonable to choose this point as a boundary of a segment-integration as the corresponding divergence is not regularized. However, such a choice turns out to be possible if (probably, after some intermediate integrations) an additional factor of the type $x_{i}^{-k \epsilon}$, where $k$ is integer, appears.

\footnotetext{
${ }^{2}$ Examples are known (see, e.g., Ref. [6]) where for master integrals some of the indices are equal to two, and not only to zero and one. However, in these situation, one can switch to another set of master integrals corresponding to indices equal to zero or a negative integer value.
} 
The basic polynomial in Eq. (77) is given by [1]

$$
P(\underline{x})=\operatorname{det}\left(\sum_{a=1}^{N} \tilde{A}_{a}^{i j}\left(x_{a}+m_{a}\right)\right),
$$

where the determinant is taken with respect to the indices $i$ and $j$. Thus, $P$ is a homogeneous polynomial of degree $h$ in the variables $x_{i}$ and the masses. The matrix $\tilde{A}_{a}^{i j}$ in Eq. (8) is defined as follows: $A_{a}^{i j}$ as introduced in Eq. (2) is defined for $i \geq j$. Let us then consider the quadratic $N \times N$ matrix $A$ (with $N=h(h+1) / 2$ ), where the first index is labeled by pairs $(i, j)$ with $i \geq j$, and the second index is $a$. The corresponding inverse matrix $\left(A^{-1}\right)_{a}^{i j}$ (where again $i \geq j$ ) satisfies

$$
\sum_{a=1}^{N} A_{a}^{i j}\left(A^{-1}\right)_{a}^{i^{\prime} j^{\prime}}=\delta_{i i^{\prime}} \delta_{j j^{\prime}} .
$$

Now $\tilde{A}_{a}^{i j}$ is defined for all $i$ and $j$ as the symmetrical extension of $\left(A^{-1}\right)_{a}^{i j}$.

As was demonstrated in [1, the representation (7) satisfies Eq. (3), provided one can use IBP in the parametric integrals. We should remark that the overall normalization in Eq. (7) is not fixed in advance but is adjusted after the construction of the coefficient functions, as a virtue of Eq. (6). For the same reason, the basic polynomial is determined up to a factor which is independent of the variables $x_{i}$.

General Feynman integrals can be reduced to the vacuum case [1, 10]. In case an external momentum is reduced to a mass shell, $p_{i}^{2}=m_{i}^{2}$ (where the mass $m_{i}$ is one of the internal masses of the diagram), one includes into the procedure all the terms of the formal Taylor expansion in $p_{i}^{2}$ at $p_{i}^{2}=m_{i}^{2}$. In the case of a general external momentum squared one can still consider similar terms of the Taylor expansion in $p_{i}^{2}-M_{i}^{2}$ where, this time, $M_{i}$ is not equal to some of the internal masses. This Taylor expansion is indeed a Taylor series (rather than a formal series) because the point $p_{i}^{2}=M_{i}^{2}$ is not singular. In this situation, one is usually interested only in the value at $p_{i}^{2}=M_{i}^{2}$ and not in the derivatives at this general point. Thus for the corresponding Feynman integrals one considers only the value $n_{i}=1$ of the corresponding index $n_{i}$. The transition to a vacuum problem effectively increases the number of loops which enters the exponent of the basic polynomial $P$ in Eq. (77) where the loop number $h$ refers to the corresponding vacuum Feynman graph. For example, in a recursive problem for a class of propagator diagrams, the transition to the corresponding vacuum problem, which is obtained after gluing the external lines, increases the loop number by one. We would like to stress that this translation to the level of vacuum Feynman integrals is not necessarily accompanied by a corresponding vacuum graph. However, in the language of Feynman integrals defined through Eq. (11) such a description is always possible.

It should be mentioned that although Eq. (77) has been applied to solve IBP recurrence relations in some physical situations [11, 12] no instructions how to apply this representation are available in the literature.

The goal of our paper is twofold. First we shall describe how the parametric representation of Eq. (7) can be used to characterize the master integrals and to construct an 
algorithm for evaluating the coefficients of these master integrals. Second, we shall choose several typical examples to exemplify the method. As a non-trivial two-loop example, we discuss in detail the class of diagrams appearing in the calculation of the two-loop heavy quark potential. The main motivation for this is that in Refs. [13, 14] no complete procedure has been presented but only the necessary Feynman integrals were calculated. A reduction procedure is described in the thesis [15] which is based on IBP accompanied by the method developed in 6]. In Ref. [16, where $1 /\left(m_{q} r^{2}\right)$ corrections to the potential were evaluated, a standard approach to solve the IBP relations has been used and an algorithm has been developed to evaluate the adequate subclass of the diagrams. In this paper we present a complete procedure for evaluating a general diagram of this class which is certainly useful if higher dimensional operators are considered within the framework of non-relativistic QCD.

The outline of this paper is as follows: in the next section, we discuss the identification of the master integrals and present general prescriptions for applying Eq. (7). In Section 3 , we discuss the one- and two-loop examples and, finally, in Section 4 we present our conclusions.

\section{Classification of master integrals and construction of coefficient functions}

In this section we present practical prescriptions to perform a reduction procedure with the help of Eq. (77). We shall assume that any Feynman integral can be decomposed according to Eqs. (5) and (6). The method decomposes into two parts: the classification of the master integrals and the construction of the coefficient functions.

\subsection{Identifying master integrals}

Let us in a first step consider the integrals where the indices corresponding to irreducible numerators are set to zero. The other indices we allow to be either zero or one. In the following we nullify all scaleless integrals (e.g., massless tadpoles) which is natural within the framework of dimensional regularization.

Before analyzing the remaining non-zero integrals with the indices one and zero, let us remember that the goal of every known reduction is to reduce some of the indices to zero. Indeed, experience tells us that a master integral $F\left(\underline{n}_{i}\right)$ never appears in the reduction of a given Feynman integral $F(\underline{n})$ if $n_{j} \leq 0$ and $n_{i j}>0$. For this reason we adopt the following natural condition for the coefficient function $c_{i}(\underline{n})$ of $F\left(\underline{n}_{i}\right)$ :

$$
c_{i}(\underline{n})=0 \quad \text { if } \quad n_{j} \leq 0 \quad \text { and } \quad n_{i j}=1 \quad \text { for } \quad j=1, \ldots, N .
$$

This condition is easily realized in an automatic way by choosing the integration domain for $x_{j}$ in Eq. (77) as a closed contour around the origin in the complex $x_{j}$-plane, which from now on will always be implied. As a consequence, the integration over the variables $x_{j}$ in Eq. (7), where $n_{i j}=1$ in the corresponding master integral, is performed with the 
help of the Cauchy theorem and thus reduces to Taylor expansions of order $n_{j}-1$ of the integrand in $x_{j}$. Eventually, Eq. (17) results in a sum of terms of the form

$$
\int \ldots \int\left[P_{i}(\underline{x})\right]^{z-n_{d}} \prod_{j: n_{i j} \leq 0} \frac{\mathrm{d} x_{j}}{x_{j}^{n_{j}^{\prime}}},
$$

where $z=(d-h-1) / 2, n_{d}$ is a non-negative integer, $n_{j}^{\prime}$ are some integer exponents, and $P_{i}(\underline{x})$ is obtained from $P(\underline{x})$ after setting to zero all variables $x_{j}$ where $n_{i j}=1$.

Let us now analyze the remaining candidates in view of being master integrals with the help of the representation (11). If $P_{i}=0$ for a given candidate $F\left(\underline{n}_{i}\right)$ (i.e., for $\underline{n}_{i}$ with $n_{i j}=1$ or 0$)$ it is naturally to put the corresponding coefficient function to zero. In other words, such a Feynman integral is recognized as a reducible integral in the sense of the reduction problem. This is the simplest condition of reducibility. A more general condition is that any resulting integral of Eq. (11) involves an integral without scale. In this situation it is natural to prescribe a zero value for it $^{3}$ and, therefore, consider the given Feynman integral $F\left(\underline{n}_{i}\right)$ as reducible in the sense of the reduction problem and the corresponding decomposition given in Eq. (5). It can happen that a scaleless integral in Eq. (71) can either be seen immediately, i.e., an integral over a "pure" power of some variable arises, or only after some intermediate integrations.

After trying all possible combinations of zero and one we obtain a list of master integrals, which fixes the terms on the right-hand side of Eq. (5).

However, this is not all. Sometimes, in addition to a given master integral $F\left(\underline{n}_{i}\right)$ with $\underline{n}_{i}$ consisting of $n_{i j}=1$ or $n_{i j}=0$, one has to consider master integrals which differ from $F\left(\underline{n}_{i}\right)$ by some indices $n_{i j}<0$. The number of such additional master integrals is dictated by the degree of the polynomial $P_{i}$ with respect to some of the parameters $x_{j}$. In practice it turns out to be advantageous to complete the list of the master integrals in the second step where algorithms for the computation of the coefficient functions $c_{i}(\underline{n})$ are constructed.

Sometimes it is obvious that a given candidate is reducible due to other arguments like the simple application of the triangle rule. However, it turns out to be rather convenient to see this property directly by analyzing the corresponding "reduced" polynomial $P_{i}$, as this provides a useful additional check.

\subsection{Constructing coefficient functions}

The set of the master integrals with the indices zero and one as obtained in the previous Subsection is partially ordered in the natural way. We write $F\left(\underline{n}_{1}\right)<F\left(\underline{n}_{2}\right)$ if $n_{1 j} \leq n_{2 j}$ for all $j$ and the strict inequality holds at least for one index. In this way all master

\footnotetext{
${ }^{3}$ At this point we once again apply a commonly accepted agreement within dimensional regularization to put any scaleless integral to zero. Observe that such a prescription, in an extended form, is also successfully applied within the strategy of expansion by regions [5] when expanding Feynman integral in various limits of masses and momenta. Moreover scaleless integrals that are not dimensionally regularized (e.g., connected with the contribution of the potential region) are also consistently put to zero with this strategy.
} 
integrals can be grouped into so-called families which are by definition disjoint. As we will see below this ordering is crucial in the construction of the coefficient functions.

Let us start from the master integrals which have the most non-zero indices where only indices corresponding to the propagators but not to the numerators are counted. These integrals are maximal in the sense of the hierarchy defined above. For a maximal master integral $F\left(\underline{n}_{i}\right)$, the corresponding coefficient function $c_{i}(\underline{n})$ in Eq. (15) is replaced by the corresponding parametric integral (7D) which results in a sum of terms as given in Eq. (111) according to our agreement of choosing integration contours for the variables associated with the indices equal to one. We want to stress that only those integrations over $x_{i}$ are non-trivial where the corresponding index of the master integral is zero. Experience shows that these remaining integration can be performed in terms of gamma functions, for the general integer values of the indices. ${ }^{4}$ At this point one applies Eq. (6) to normalize the resulting expression.

Let us now suppose that we are dealing with a master integral which is not maximal, i.e., we have two master integrals which fulfill the hierarchy $F\left(\underline{n}_{2}\right)<F\left(\underline{n}_{1}\right)$. This means that if $n_{2 i}=1$ we have also $n_{1 i}=1$. To construct an algorithm for the coefficient function $c_{2}(\underline{n})$ we start with the case of negative indices $n_{j}$ for those indices $j$ where $n_{1 i}=1$ as in this case we have $c_{1}(\underline{n})=0$. Experience shows that the integrations for $c_{2}(\underline{n})$ result in ratios of gamma functions which in particular can be used to obtain $c_{2}\left(\underline{n}_{2}\right)=1$. In a next step one considers the case $n_{j}>0$. Then the corresponding parametric representation (17) usually leads to integrals (cf. Eq. (11)) which cannot be evaluated in gamma functions. ${ }^{5}$ Thus at first sight it looks hopeless to achieve that the coefficient functions have to be rational functions of $d$ (and eventually other dimensionful quantities). The way out of this problem is as follows: actually we have to look for an expression for the coefficient function $c_{2}(\underline{n})$ which is a linear combination of $c_{1}(\underline{n})$ and the representation (7) for $c_{2}(\underline{n})$. Denoting the latter by $c_{2}^{0}(\underline{n})$ one has

$$
c_{2}(\underline{n})=c_{2}^{0}(\underline{n})+A c_{1}(\underline{n}),
$$

where the constant $A$ is determined by the condition $c_{2}\left(\underline{n}_{1}\right)=0$ (cf. Eq. (지) ) and is thus given by

$$
A=-c_{2}^{0}\left(\underline{n}_{1}\right) .
$$

At this stage it turns out that it is advantageous to use IBP applied to the parametric integrals of Eq. (11). Note that the complexity is significantly reduced as compared to the original integral simply because there are much less variables involved. The basic idea is to express any given parametric integral in terms of auxiliary (parametric) master integrals and expressions which are straightforwardly evaluated in gamma functions. The dependence on the new auxiliary master integrals has to drop out in order to guarantee that the coefficient functions are rational functions of $d$ (and eventually the other dimensionful parameters). Such a cancellation turns to be a good check of the algorithm. This

\footnotetext{
${ }^{4}$ Typically, integrals involving the Euler beta function arise as we will see in the examples discussed in Section 3

${ }^{5}$ However, sometimes this is possible as we will see in the examples below.
} 
is similar to the cancellation of spurious poles when applying general prescriptions for asymptotic expansion of Feynman integrals in various limits of momenta and masses [5]. In our particular example of a hierarchy of two master integrals one presumably only needs one auxiliary parametric master integral.

For a generic tower of master integrals the construction and cancellation of the auxiliary master integrals goes along the same lines. When constructing the corresponding coefficient function one uses a linear combination of the coefficient under consideration and all coefficients of the higher master integrals. The constants are determined with the help of Eq. (6). Explicit examples can be found in Section 3

Let us stress again that in general it is rather simple to compute the coefficient function of a maximal master integral as it has many indices equal to one. On the other hand, following the procedure described above, it is possible to either reduce the indices of the coefficient of a non-maximal master integral in order to make an evaluation in $\Gamma$ functions possible, or relate it to the coefficient functions of higher master integrals. This is achied with the help of IBP applied to the parametric integrals over the $x$-parameters.

At this point it may happen that the initial list of the master integrals obtained in Section 2.1 is not sufficient and has to be extended by additional master integrals where some of the indices are negative. This necessity is connected to the type of solution of the recursive problem for the auxiliary parametric integrals and thus to the basic polynomial $P_{i}(\underline{x})$.

We consider the construction of a coefficient functions as completed if for given indices an algorithm (usually realized on a computer) for its evaluation is provided. However, in simple situations, one can even obtain explicit expressions for the coefficients as functions of the indices.

After we have obtained the list of the master integrals it is reasonable to check whether there are identical expressions among them, although they all have appeared in a different way. If some of the master integrals within one family are equal simply because the corresponding integrals are identical, e.g., due to some symmetry, it is natural to consider one master integral instead. The corresponding coefficient function is the sum of the coefficient functions of the original integrals. From the mathematical point of view, the best solution would be to achieve the absolute minimal number of the master integrals in Eq. (51). However, for practical purposes, this is not very important.

Finally let us note that it is possible to identify an integral as master and then immediately construct an algorithm for the corresponding coefficient function, rather than strictly decompose the procedure into the two steps as described above. 


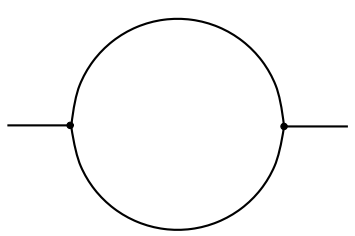

(a)

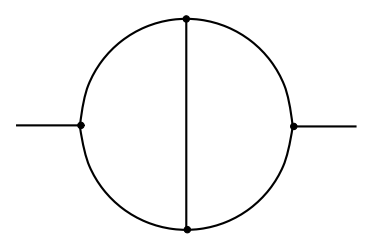

(b)

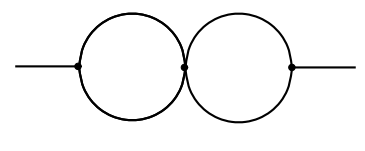

(c)

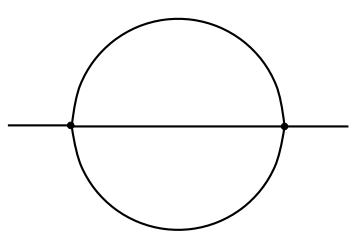

(d)

Figure 1: Feynman diagrams for one- and two-loop massless propagator ((a) and (b)). At two-loop order there are two master integrals which are shown in (c) and (d).

\section{One- and two-loop examples}

\subsection{One-loop propagator diagram}

Let us start with the simple example of a massless one-loop diagram with one external momentum (a so-called propagator diagram) which is given by (see also Fig. 1(a))

$$
F\left(n_{1}, n_{2}\right)=\int \frac{\mathrm{d}^{d} k}{\left(k^{2}\right)^{n_{1}}\left[(k-q)^{2}\right]^{n_{2}}},
$$

where the usual prescriptions like $k^{2}=k^{2}+i 0$, etc. are implied. According to [1], the transition to the corresponding vacuum problem reduces to adding a new propagator, $1 /\left(q^{2}-s\right)$, with some mass squared $s$. Furthermore one has $h=2$. We want to consider the value of our diagram at some general point and are not interested in higher terms of the Taylor expansion in $q^{2}$. Therefore we consider only the index $n_{3}=1$ corresponding to the additional propagator. The integration contour for the corresponding variable $x_{3}$ is taken as a Cauchy contour around the origin. Thus we write down the corresponding basic polynomial (8) and reduce it by setting $x_{3}=0$. After that the resulting basic polynomial reads

$$
P\left(x_{1}, x_{2}\right)=\left(q^{2}\right)^{2}-2 q^{2}\left(x_{1}+x_{2}\right)+\left(x_{1}-x_{2}\right)^{2} .
$$

The only possible candidate for a master integral is $F(1,1)$ because we obtain massless tadpoles when we put one of the indices to zero. We have

$$
I_{1}=F(1,1)=i \pi^{d / 2}\left(-q^{2}\right)^{d / 2-2} \frac{\Gamma(2-d / 2) \Gamma^{2}(d / 2-1)}{\Gamma(d-2)} .
$$

The corresponding coefficient function is easily obtained from Eq. (17) with the help of the Cauchy theorem

$$
c_{1}\left(n_{1}, n_{2}\right)=\left.N_{1} \frac{1}{\left(n_{1}-1\right) !}\left(\frac{\partial}{\partial x_{1}}\right)^{n_{1}-1} \frac{1}{\left(n_{2}-1\right) !}\left(\frac{\partial}{\partial x_{2}}\right)^{n_{2}-1}\left[P\left(x_{1}, x_{2}\right)\right]^{(d-3) / 2}\right|_{x_{i}=0}
$$


where $N_{1}$ is a ( $d$-dependent) normalization factor introduced in order to fulfill Eq. (6). For our choice of $P\left(x_{1}, x_{2}\right)$ it reads

$$
N_{1}=\left(q^{2}\right)^{(d-3)}
$$

It is straightforward to implement Eq. (17) on a computer. The result agrees with the known one which in this case, of course, is trivially obtained by a straightforward Feynman parameterization.

\subsection{Two-loop propagator diagram}

The situation becomes slightly more complicated at two-loop level where the Feynman integral is given by (cf. Fig. 1(b))

$$
F(\underline{n})=\int \frac{\mathrm{d}^{d} k}{\left(k^{2}\right)^{n_{1}}\left(l^{2}\right)^{n_{2}}\left[(k-q)^{2}\right]^{n_{3}}\left[(l-q)^{2}\right]^{n_{4}}\left[(k-l)^{2}\right]^{n_{5}}} .
$$

The transition to vacuum integrals is performed in analogy to the previous one-loop case by introducing one more propagator connected with the external invariant $q^{2}$ and $h=3$ (see also the next example for more details). As before, we set the corresponding $x$-parameter to zero and obtain the following basic polynomial

$$
\begin{aligned}
P\left(x_{1}, \ldots, x_{5}\right)= & -x_{1} x_{2} x_{3}+x_{2}^{2} x_{3}+x_{2} x_{3}^{2}+x_{1}^{2} x_{4}-x_{1} x_{2} x_{4}-x_{1} x_{3} x_{4}-x_{2} x_{3} x_{4}+x_{1} x_{4}^{2} \\
& +x_{1} x_{2} x_{5}-x_{2} x_{3} x_{5}-x_{1} x_{4} x_{5}+x_{3} x_{4} x_{5}+q^{2}\left[-x_{1} x_{3}+x_{2} x_{3}+x_{1} x_{4}\right. \\
& \left.-x_{2} x_{4}+x_{1} x_{5}+x_{2} x_{5}+x_{3} x_{5}+x_{4} x_{5}-x_{5}^{2}\right]+\left(q^{2}\right)^{2} x_{5} .
\end{aligned}
$$

An analysis of the integrals with indices equal to zero leads to two master integrals shown in Fig. 1(c) and (d)

$$
\begin{aligned}
& I_{1}=F(1,1,1,1,0)=\left(i \pi^{d / 2}\right)^{2}\left(-q^{2}\right)^{d-4} \frac{\Gamma^{2}(2-d / 2) \Gamma^{4}(d / 2-1)}{\Gamma^{2}(d-2)} \\
& I_{2}=F(0,1,1,0,1)=F(1,0,0,1,1)=-\left(i \pi^{d / 2}\right)^{2}\left(-q^{2}\right)^{d-3} \frac{\Gamma(3-d) \Gamma^{3}(d / 2-1)}{\Gamma(3 d / 2-3)}
\end{aligned}
$$

where the second one appears in two ways. As far as the coefficient functions are concerned the integration over those $x_{i}$ with index " 1 " in the corresponding master integral is trivially obtained through differentiation of $P(\underline{x})$. In the case of $c_{1}$ this leads to the following remaining integral

$$
I_{h}^{(1)}(\alpha, \beta)=\int_{0}^{q^{2}} \mathrm{~d} x_{5} x_{5}^{\alpha}\left(q^{2}-x_{5}\right)^{\beta}=\left(q^{2}\right)^{\alpha+\beta+1} \frac{\Gamma(\alpha+1) \Gamma(\beta+1)}{\Gamma(\alpha+\beta+2)} .
$$

Here and in the following $\alpha$ and $\beta$ depend on the dimension $d$ whereas $k$ represent integer indices. On the other hand, for $c_{2}$ one has to solve a two-dimensional integral over $x_{1}$ and 


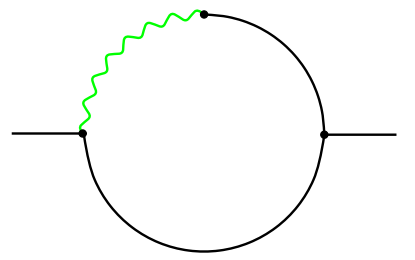

(a)

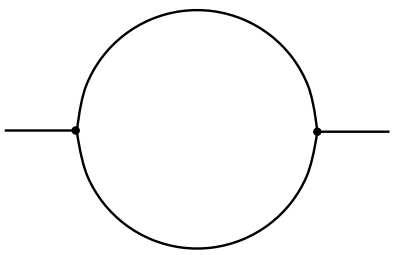

(b)

Figure 2: (a) Generic Feynman diagram needed for the one-loop heavy quark potential. There two master integrals: one corresponds to the very diagram with all indices equal to one and the second one is shown (b).

$x_{4}$ which is calculated as follows

$$
\begin{aligned}
I_{h}^{(2)}\left(\alpha_{1}, \alpha_{4}, \beta\right) & =\int_{0}^{\infty} \mathrm{d} x_{1} \mathrm{~d} x_{4} x_{1}^{\alpha_{1}} x_{4}^{\alpha_{4}}\left(q^{2}+x_{1}+x_{4}\right)^{\beta} \\
& =\left(q^{2}\right)^{\alpha_{1}+\alpha_{4}+\beta+2} \frac{\Gamma\left(\alpha_{1}+1\right) \Gamma\left(\alpha_{4}+1\right) \Gamma\left(-\alpha_{1}-\alpha_{4}-\beta-2\right)}{\Gamma(-\beta)} .
\end{aligned}
$$

Again a small computer program takes over the differentiation and integration parts and finally results to the two-loop integral

$$
\begin{aligned}
F\left(n_{1}, n_{2}, n_{3}, n_{4}, n_{5}\right)= & c_{1}\left(n_{1}, n_{2}, n_{3}, n_{4}, n_{5}\right) I_{1} \\
& +\left[c_{2}\left(n_{1}, n_{2}, n_{3}, n_{4}, n_{5}\right)+c_{2}\left(n_{2}, n_{1}, n_{4}, n_{3}, n_{5}\right)\right] I_{2},
\end{aligned}
$$

in agreement with the usual approach based on IBP [3].

\subsection{One-loop diagram for the heavy quark potential}

The integrals studied in this and the next subsection are useful in the context of nonrelativistic QCD (NRQCD) where in addition to relativistic propagators (for gluons and light quarks) also non-relativistic ones (for heavy quarks) appear. The general one-loop integral is shown in Fig. 2 and has the form

$$
F\left(n_{1}, n_{2}, n_{3}\right)=\int \frac{\mathrm{d}^{d} k}{\left(k^{2}\right)^{n_{1}}\left[(k-q)^{2}\right]^{n_{2}}(v \cdot k)^{n_{3}}},
$$

with $v \cdot k$ understood as $v \cdot k+i 0$. Here $q$ is the external momentum and $v$ is a constant vector which fulfills the condition $v \cdot q=0$.

When turning to the corresponding vacuum problem we consider, in addition to $k^{2}$, $q \cdot k$ and $v \cdot k$, three extra kinematical invariants, $q^{2}, v \cdot q, v^{2}$, which play the role of inverse auxiliary propagators. The latter are obtained after interpreting the expression in Eq. (25) as a three-point function with external momenta $q$ and $v$ and closing the three external lines in a new vertex. This formally leads to a three-loop vacuum problem and thus to $h=3$. 
In the notation of Eq. (11) we have $D_{1}=k^{2}, D_{2}=(k-q)^{2}, D_{3}=k \cdot v+v^{2}, D_{4}=v^{2}, D_{5}=$ $q^{2}$ and $D_{6}=(q+v)^{2}$ which defines the matrix $A$ according to Eq. (2). The symmetrical extension of the corresponding inverse matrix leads to (after identifying the six invariants with $\left.x_{1}, \ldots, x_{6}\right)$

$$
\left(\begin{array}{ccc}
x_{1} & \left(x_{1}-x_{2}+x_{5}\right) / 2 & x_{3}-x_{4} \\
\left(x_{1}-x_{2}+x_{5}\right) / 2 & x_{5} & \left(-x_{4}-x_{5}+x_{6}\right) / 2 \\
x_{3}-x_{4} & \left(-x_{4}-x_{5}+x_{6}\right) / 2 & x_{4}
\end{array}\right) .
$$

The basic polynomial $P$ is obtained from the determinant of this matrix with the help of Eq. (8). Moreover, we shift the variables $x_{i}$ by the corresponding effective masses: $x_{3} \rightarrow x_{3}+v^{2}, x_{4} \rightarrow x_{4}+v^{2}, x_{5} \rightarrow x_{5}+q^{2}, x_{6} \rightarrow x_{6}+(q+v)^{2}$. Note, that as in the previous examples we are not interested in higher order Taylor coefficients of the additional kinematical invariants. Thus we can reduce the basic polynomial by setting $x_{4}=x_{5}=$ $x_{6}=0$ and finally obtain

$$
P\left(x_{1}, x_{2}, x_{3}\right)=\left(q^{2}\right)^{2} v^{2}+v^{2}\left(x_{1}-x_{2}\right)^{2}+2 q^{2}\left[v^{2}\left(x_{1}+x_{2}\right)-2 x_{3}^{2}\right],
$$

which leads to the two master integrals

$$
\begin{aligned}
& I_{1}=F(1,1,1)=-i \pi^{d / 2} \frac{\left(-q^{2}\right)^{d / 2-5 / 2} \sqrt{\pi}}{v} \frac{\Gamma(5 / 2-d / 2) \Gamma^{2}(d / 2-3 / 2)}{\Gamma(d-3)}, \\
& I_{2}=F(1,1,0)=i \pi^{d / 2}\left(-q^{2}\right)^{d / 2-2} \frac{\Gamma(2-d / 2) \Gamma^{2}(d / 2-1)}{\Gamma(d-2)} .
\end{aligned}
$$

Since for $I_{1}$ all indices are one the coefficient function $c_{1}$ is obtained in analogy to the one of Eq. (17). In the case of $c_{2}$ the indices $n_{1}$ and $n_{2}$ are treated with the help of differentiations which leads to the following type of integral for $x_{3}$

$$
I_{h}^{(3)}\left(k_{3}, \alpha\right)=\int_{-a}^{a} \mathrm{~d} x_{3} x_{3}^{k_{3}}\left(a^{2}-x_{3}^{2}\right)^{\alpha} .
$$

Here $k_{3}$ is an integer but $\alpha$ depends on $d . I_{h}^{(3)}(k, \alpha)$ can be interpreted as a principal value integral leading to

$$
I_{h}^{(3)}(k, \alpha)=\left\{\begin{array}{ll}
\left(a^{2}\right)^{\alpha+k / 2+1 / 2} \frac{\Gamma(k / 2+1 / 2) \Gamma(\alpha+1)}{\Gamma(\alpha+k / 2+3 / 2)} & \text { for } k \text { even } \\
0 & \text { for } k \text { odd }
\end{array} .\right.
$$

The final result

$$
F\left(n_{1}, n_{2}, n_{3}\right)=c_{1}\left(n_{1}, n_{2}, n_{3}\right) I_{1}+c_{2}\left(n_{1}, n_{2}, n_{3}\right) I_{2}
$$

agrees with the explicit analytical result (see, e.g., Ref. [15]). 


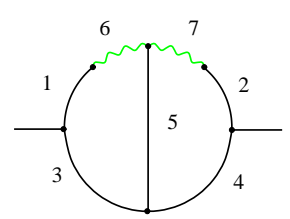

(a)



(b)

Figure 3: Feynman diagrams corresponding to case A (a) and case B (b).

\subsection{Two-loop diagrams for the heavy quark potential}

At two-loop order two classes of Feynman integrals are needed which we will refer to as case $\mathrm{A}$ and case $\mathrm{B}$

$$
\begin{aligned}
& F_{A}(\underline{n})=\iint \frac{\mathrm{d}^{d} k \mathrm{~d}^{d} l}{\left(k^{2}\right)^{n_{1}}\left(l^{2}\right)^{n_{2}}\left[(k-q)^{2}\right]^{n_{3}}\left[(l-q)^{2}\right]^{n_{4}}\left[(k-l)^{2}\right]^{n_{5}}(v \cdot k)^{n_{6}}(v \cdot l)^{n_{7}}}, \\
& F_{B}(\underline{n})=\iint \frac{\mathrm{d}^{d} k \mathrm{~d}^{d} l}{\left(k^{2}\right)^{n_{1}}\left(l^{2}\right)^{n_{2}}\left[(k-q)^{2}\right]^{n_{3}}\left[(l-q)^{2}\right]^{n_{4}}\left[(k-l)^{2}\right]^{n_{5}}(v \cdot k)^{n_{6}}[v \cdot(k-l)]^{n_{7}}} .
\end{aligned}
$$

They are shown in Fig. 3. The corresponding basic polynomials read

$$
\begin{aligned}
P_{A}\left(x_{1}, \ldots, x_{7}\right)= & -\left[x_{2} x_{6}-x_{4} x_{6}+\left(-x_{1}+x_{3}\right) x_{7}\right]^{2}+v^{2}\left\{x_{1}^{2} x_{4}+x_{3}\left(x_{2}^{2}+x_{2}\left(x_{3}-x_{4}-x_{5}\right)\right.\right. \\
& \left.\left.+x_{4} x_{5}\right)-x_{1}\left[x_{2}\left(x_{3}+x_{4}-x_{5}\right)+x_{4}\left(x_{3}-x_{4}+x_{5}\right)\right]\right\} \\
& +\left(q^{2}\right)^{2}\left[v^{2} x_{5}-\left(x_{6}-x_{7}\right)^{2}\right]+q^{2}\left\{v ^ { 2 } \left[\left(x_{3}+x_{4}-x_{5}\right) x_{5}+x_{2}\left(x_{3}-x_{4}+x_{5}\right)\right.\right. \\
& \left.+x_{1}\left(-x_{3}+x_{4}+x_{5}\right)\right]+2\left[x_{2} x_{6}\left(-x_{6}+x_{7}\right)+x_{4} x_{6}\left(-x_{6}+x_{7}\right)\right. \\
& \left.\left.+x_{7}\left(x_{1} x_{6}+x_{3} x_{6}-2 x_{5} x_{6}-x_{1} x_{7}-x_{3} x_{7}\right)\right]\right\} \\
P_{B}\left(x_{1}, \ldots, x_{7}\right)= & P_{A}\left(x_{1}, x_{2}, x_{3}, x_{4}, x_{5}, x_{6}, x_{6}-x_{7}\right) .
\end{aligned}
$$

The application of the procedures described in Section 2 to case A leads to the following families of master integrals which are also pictured in Fig. 4. As far as the notation is concerned the first index labels the different master integrals. In case the master integrals are equal we introduce a second index for further specification. An additional subscript " $a "$ is added for those master integrals where one index is " $-1 "$.

- Family A1. One has four master integrals which obey the following hierarchy: $I_{1}>\left\{I_{21}, I_{22}\right\}>I_{3}$ with

$$
\begin{aligned}
I_{1} & =F_{A}(1,1,1,1,0,1,1), \\
I_{21} & =F_{A}(1,1,1,1,0,0,1), \\
I_{22} & =F_{A}(1,1,1,1,0,1,0), \\
I_{3} & =F_{A}(1,1,1,1,0,0,0) .
\end{aligned}
$$

- Family A2. One has four master integrals which obey the following hierarchy: $I_{51}>\left\{I_{71}, I_{81}\right\}>I_{41}$ with

$$
I_{51}=F_{A}(1,0,0,1,1,1,1),
$$


Family A1:
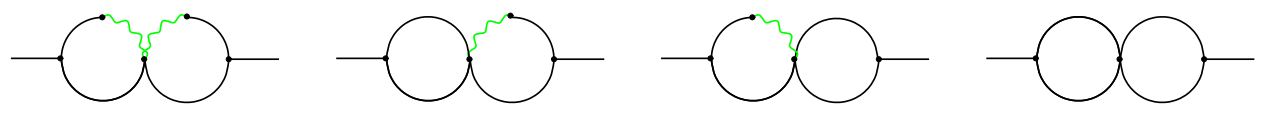

$$
I_{1}
$$

$I_{21}$

$I_{22}$

$I_{3}$

Family A2:


Family A4:

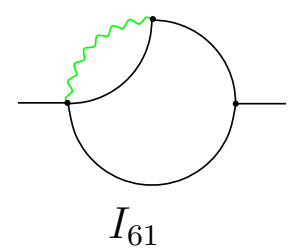

Figure 4: Feynman diagrams corresponding to the master integrals of case A. In addition to $I_{61}$ there is also a master integral $\left(I_{61 a}\right)$ containing an irreducible numerator (see text).

$$
\begin{aligned}
& I_{71}=F_{A}(1,0,0,1,1,0,1), \\
& I_{81}=F_{A}(1,0,0,1,1,1,0), \\
& I_{41}=F_{A}(1,0,0,1,1,0,0) .
\end{aligned}
$$

- Family A3 is symmetrical to Family A2 with respect to $1 \leftrightarrow 2,3 \leftrightarrow 4,6 \leftrightarrow 7$. It contains the master integrals $I_{52}, I_{72}, I_{82}$ and $I_{42}$.

- Family A4 contains the master integrals

$$
\begin{aligned}
I_{61} & =F_{A}(0,1,0,1,1,1,0), \\
I_{61 a} & =F_{A}(0,1,0,1,1,1,-1) .
\end{aligned}
$$

- Family A5 is symmetrical to Family A4 with respect to $1 \leftrightarrow 2,3 \leftrightarrow 4,6 \leftrightarrow 7$. It contains the master integrals $I_{62}$ and $I_{62 a}$.

As expected, the master integrals of the massless scalar two-loop diagram discussed in Section 3.2 also appear in the above list.

As has already become clear from the examples discussed so far, one expects the appearance of complicated expressions for the coefficient functions of the simple master integrals. Indeed, in the case of the coefficient function $c_{1}$ six out of seven indices can be treated with the help of differentiations and the remaining one-dimensional integral is easily solved with the help of $I_{h}^{(1)}$ given in Eq. (22). The situation is similar for $c_{22}$ 
(and $c_{21}$ which can be obtained by exploiting the symmetry) where the remaining two-fold integration over $x_{7}$ and $x_{5}$ is performed with the help of

$$
\begin{aligned}
I_{h}^{(4)}\left(k, \alpha_{1}, \alpha_{2}\right) & =\int_{a}^{b} \mathrm{~d} x x^{k}(x-a)^{\alpha_{1}}(b-x)^{\alpha_{2}} \\
& =\sum_{r=0}^{k} a^{k-r}(b-a)^{\alpha_{1}+\alpha_{2}+r+1} \frac{k !}{(k-r) ! r !} \frac{\Gamma\left(1+\alpha_{2}\right) \Gamma\left(1+\alpha_{1}+r\right)}{\Gamma\left(\alpha_{1}+\alpha_{2}+r+2\right)} .
\end{aligned}
$$

and Eqs. (29) and (30), respectively. In principle, instead of performing the $x_{5}$ integration one could also introduce an auxiliary master integral as a result of a two-dimensional $\left(x_{5}\right.$ and the dimension $d$ ) recursion problem. This master integral would cancel after considering the proper linear combination with $c_{1}$ as described around Eq. (12).

For the coefficient $c_{3}$ there are three non-trivial integrations $\left(x_{5}, x_{6}, x_{7}\right)$ left. In case one of the indices $n_{5}, n_{6}$ or $n_{7}$ is less or equal to zero one can use various combinations of the auxiliary integrals $I_{h}^{(i)}(i=1, \ldots, 4)$ listed above. Thereby it is advantageous to perform the integration corresponding to the negative index first. If, on the contrary, $n_{5}$, $n_{6}$ and $n_{7}$ are positive an immediate integration seems not to be possible. However, from the corresponding three-parametric integral representation it is simple to derive recurrence relations which shift at least one of the indices to zero, eventually at the cost of increasing the dimension. The latter does not constitute a problem since the whole formulation of our procedure is in $d$ dimensions. Thus, also in this case the integration can be performed in terms of $\Gamma$ functions. In principle one could be forced to introduce three auxiliary master integrals and build the proper linear combinations with $c_{1}, c_{21}$ and $c_{22}$. However, it turns out that the corresponding constants, $c_{3}\left(\underline{n}_{i}\right)(i=1,2,3)$ are zero.

For the coefficient function $c_{51}$ only two non-trivial integrations over $x_{2}$ and $x_{3}$ are involved which can be performed with the help of Eq. (23).

The situation is similar for $c_{71}$. It is convenient to perform in a first step the $x_{6}$ integration with the help of $I_{h}^{(3)}$ of Eq. (29). For the remaining integration over $x_{2}$ and $x_{3}$ the formula

$$
\begin{aligned}
I_{h}^{(5)}\left(k, \alpha_{2}, \alpha_{3}, \beta\right)= & \int_{0}^{\infty} \mathrm{d} x_{2} \mathrm{~d} x_{3} x_{2}^{\alpha_{2}} x_{3}^{\alpha_{3}}\left(q^{2}+x_{2}+x_{3}\right)^{\beta}\left(q^{2}+x_{2}\right)^{k} \\
= & \left(q^{2}\right)^{\alpha_{2}+\alpha_{3}+\beta+k+2} \frac{\Gamma\left(\alpha_{2}+1\right) \Gamma\left(\alpha_{3}+1\right) \Gamma\left(-\alpha_{2}-\alpha_{3}-\beta-k-2\right)}{\Gamma(-\beta)} \\
& \times \frac{\Gamma\left(-\alpha_{3}-\beta-1\right)}{\Gamma\left(-\alpha_{3}-\beta-k-1\right)},
\end{aligned}
$$

turns out to be very useful. In principle, the complete construction of $c_{71}$ requires the proper linear combination with $c_{51}$. However, since we have $c_{71}\left(\underline{n}_{51}\right)=0$ this is not necessary. For $c_{81}$ no separate calculation is necessary as the symmetry of the basic polynomial can be exploited and one has $c_{81}\left(n_{1}, n_{2}, n_{3}, n_{4}, n_{5}, n_{6}, n_{7}\right)=c_{71}\left(n_{4}, n_{3}, n_{2}, n_{1}, n_{5}, n_{7}, n_{6}\right)$.

The most complicated coefficient function is certainly $c_{41}$ since there are four nontrivial integrations over $x_{2}, x_{3}, x_{6}$ and $x_{7}$ left. If $n_{6}$ or $n_{7}$ are less than or equal to zero the integrations can be performed in terms of $\Gamma$ functions with the help of the formulae 
provided above. However, for $n_{6} \geq 1$ and $n_{7} \geq 1$ this is not possible. In this case the idea is to use IBP in order to reduce the four-parametric integral representation

$$
I_{41}^{A, \operatorname{aux}}\left(n_{2}, n_{3}, n_{6}, n_{7}, n_{d}\right)=\int \ldots \int\left[P_{41}\left(x_{2}, x_{3}, x_{6}, x_{7}\right)\right]^{z-n_{d}} \frac{\mathrm{d} x_{2} \mathrm{~d} x_{3} \mathrm{~d} x_{6} \mathrm{~d} x_{7}}{x_{2}^{n_{2}} x_{3}^{n_{3}} x_{6}^{n_{6}} x_{7}^{n_{7}}}
$$

(with $z=(d-h-1) / 2=d / 2-5 / 2)$ to the auxiliary master integral $I_{41}^{A \text { aux }}(1,1,1,1,0)$. $P_{41}$ is obtained from $P_{A}$ by setting $x_{1}, x_{4}$ and $x_{5}$ to zero. We should stress that the corresponding recurrence procedure is significantly simpler than the original one which involves seven denominators. Furthermore, if during the recursion either $n_{6}$ or $n_{7}$ becomes negative the corresponding expressions can immediately be expressed in terms of $\Gamma$ functions. The five IBP relations which are useful for the reduction to $I_{41}^{A \text {, aux }}(1,1,1,1,0)$ can be obtained by either differentiating the integrand with respect to $x_{i}(i=2,3,6,7)$ or by writing $P_{41}^{z-n_{d}}=P_{41}^{z-n_{d}-1} P_{41}$ and inserting the explicit result for the last factor. The proper combination of these relations leads to new ones which allows the following steps to be performed in an automatic way:

1. Reduce $n_{6}$ and $n_{7}$ to one.

2. Reduce $n_{2}, n_{3}>0$ to $n_{2}, n_{3} \leq 0$.

3. Use IBP recurrence relations to obtain $n_{2}=n_{3}$.

4. Reduce $n_{2}=n_{3}<0$ to $n_{2}=n_{3}=0$.

5. Adjust the dimension, i.e., reduce $n_{d}$ to zero.

A simple relation transforms $I_{41}^{A \text {,aux }}(0,0,1,1,0)$ to $I_{41}^{A \text {,aux }}(1,1,1,1,0)$. At this point one constructs the final coefficient function $c_{41}$ by considering the linear combination with $c_{51}$, $c_{71}$ and $c_{81}$. Since one has $c_{41}\left(\underline{n}_{71}\right)=c_{41}\left(\underline{n}_{81}\right)=0$ we are left with

$$
c_{41}(\underline{n})=c_{41}^{0}(\underline{n})-c_{41}^{0}\left(\underline{n}_{51}\right) c_{51}(\underline{n}),
$$

where

$$
\begin{aligned}
c_{41}^{0}\left(\underline{n}_{51}\right)= & -\frac{1}{q^{2} v^{2}} \frac{4(d-3)(3 d-14)(3 d-10)(3 d-8)}{(d-4)^{2}(3 d-13)(3 d-11)} \\
& +\frac{(d-5)^{2}}{(3 d-13)(3 d-11)}\left(q^{2}\right)^{2} I_{41}^{A, \text { aux }}(1,1,1,1,0) .
\end{aligned}
$$

In this combination the auxiliary master integral $I_{41}^{A \text {,aux }}(1,1,1,1,0)$ cancels and $c_{41}(\underline{n})$ is a rational function in $d$.

The master integral $I_{61}$ forms a family by its own. However, as the polynomial $P_{61}$ is quadratic in $x_{7}$ and thus the corresponding recurrence relation shifts $n_{7}$ only in steps of two it is necessary to introduce in addition the master integral $I_{61 a}$ where $n_{7}=-1$. The practical implementation within our method does not need this recurrence relation, 


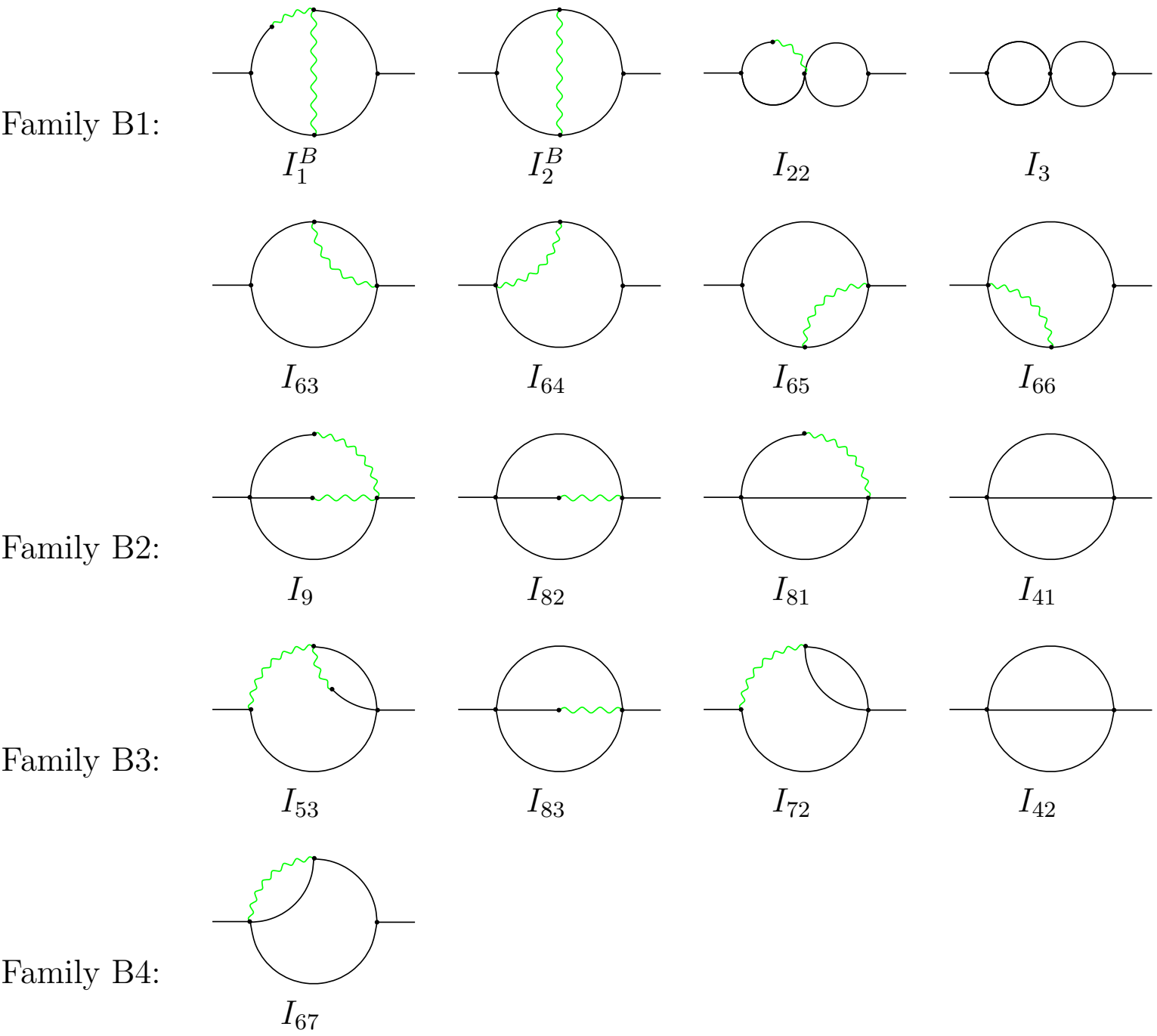

Figure 5: Feynman diagrams corresponding to the master integrals of case B. In addition to $I_{6 i}(i=3, \ldots, 7)$ there are also master integrals $\left(I_{6 i a}\right)$ containing an irreducible numerators (see text).

however, feels the necessity of introducing $I_{61 a}$. The very calculation of the coefficient function is identical for $I_{61}$ and $I_{61 a}$. For $n_{3} \leq 0$ it can be done in terms of $\Gamma$ functions with the integration order $x_{3}, x_{1}, x_{7}$. On the other hand, for $n_{3}>0$ a simple one-step relation reduces $n_{3}$ to zero.

Let us now come to case B. As one can see from Eq. (35) the basic polynomial is quite similar to the one of case A which can be used while computing the coefficient functions. However, the symmetry can only be exploited if $n_{7} \leq 0$ as for $n_{7}>0$ the factor $\left(x_{6}-x_{7}\right)$ would appear in the denominator.

Altogether there are four families which, however, show a more complicated structure than in case A. More precisely one has 
- Family B1. There are twelve master integrals which obey the hierarchies $I_{1}^{B}>$ $\left\{I_{2}^{B}, I_{22}\right\}>I_{3}$ and $I_{1}^{B}>I_{2}^{B}>\left\{I_{6 i}, I_{6 i a}\right\}(i=3,4,5,6)$ and are given by

$$
\begin{aligned}
I_{1}^{B} & =F_{B}(1,1,1,1,0,1,1), \\
I_{2}^{B} & =F_{B}(1,1,1,1,0,0,1), \\
I_{22} & =F_{B}(1,1,1,1,0,1,0), \\
I_{3} & =F_{B}(1,1,1,1,0,0,0), \\
I_{63} & =F_{B}(1,1,1,0,0,0,1), \\
I_{64} & =F_{B}(1,1,0,1,0,0,1), \\
I_{65} & =F_{B}(1,0,1,1,0,0,1), \\
I_{66} & =F_{B}(0,1,1,1,0,0,1) .
\end{aligned}
$$

Furthermore there are master integrals with $n_{6}=-1$

$$
\begin{aligned}
& I_{63 a}=F_{B}(1,1,1,0,0,-1,1), \\
& I_{64 a}=F_{B}(1,1,0,1,0,-1,1), \\
& I_{65 a}=F_{B}(1,0,1,1,0,-1,1), \\
& I_{66 a}=F_{B}(0,1,1,1,0,-1,1) .
\end{aligned}
$$

- Family B2. One has four master integrals which obey the following hierarchy: $I_{9}>$ $\left\{I_{82}, I_{81}\right\}>I_{41}$ with

$$
\begin{aligned}
I_{9} & =F_{B}(1,0,0,1,1,1,1), \\
I_{82} & =F_{B}(1,0,0,1,1,0,1), \\
I_{81} & =F_{B}(1,0,0,1,1,1,0), \\
I_{41} & =F_{B}(1,0,0,1,1,0,0) .
\end{aligned}
$$

- Family B3. Similarly to Family B2 one has four master integrals obeying the hierarchy $I_{53}>\left\{I_{83}, I_{72}\right\}>I_{42}$ with

$$
\begin{aligned}
& I_{53}=F_{B}(0,1,1,0,1,1,1), \\
& I_{83}=F_{B}(0,1,1,0,1,0,1), \\
& I_{72}=F_{B}(0,1,1,0,1,1,0), \\
& I_{42}=F_{B}(0,1,1,0,1,0,0) .
\end{aligned}
$$

- Family B4 consists of the two master integrals

$$
\begin{gathered}
I_{67}=F_{B}(0,1,0,1,1,1,0), \\
I_{67 a}=F_{B}(0,1,0,1,1,1,-1),
\end{gathered}
$$

which is similar to the Families A4 and A5 of case A. 
The construction of the coefficient functions $c_{1}^{B}, c_{2}^{B}$ and $c_{22}$ from the family B1 proceeds along the same lines as in case $\mathrm{A}$. In the case of $c_{3}$ there is a slight complication as, in contrast to case $\mathrm{A}, c_{3}\left(\underline{n}_{1}\right) \neq 0$. As a consequence an auxiliary master integral ${ }^{6}$, $I_{3}^{B \text {,aux }}(0,1,1,0)$ has to be introduced which is only canceled after considering the proper linear combination with $c_{1}$. The reduction to $I_{3}^{B \text {,aux }}(0,1,1,0)$ is straightforward.

Family B1 has four more members, $I_{63}, I_{64}, I_{65}$ and $I_{66}$, which belong to the four hierarchies $I_{1}^{B}>I_{2}^{B}>I_{6 i}(i=3,4,5,6)$. Thus, in order to obtain the coefficient functions $c_{6 i}$ one has to consider the linear combination

$$
c_{6 i}=c_{6 i}^{0}-c_{6 i}^{0}\left(\underline{n}_{1}^{B}\right) c_{1}^{B}(\underline{n})-c_{6 i}^{0}\left(\underline{n}_{2}^{B}\right) c_{2}^{B}(\underline{n}) .
$$

Let us in the following restrict the discussion to $c_{63}$ since the results for the other three coefficients can be obtained by exploiting the symmetry. $c_{63}^{0}$ is given by an integral representation of the form

$$
c_{63}^{0} \sim \int \ldots \int\left[P_{63}\left(x_{4}, x_{5}, x_{6}\right)\right]^{z-n_{d}} \frac{\mathrm{d} x_{4} \mathrm{~d} x_{5} \mathrm{~d} x_{6}}{x_{4}^{n_{4}} x_{5}^{n_{5}} x_{6}^{n_{6}}}
$$

with

$$
P_{63}=\left(q^{2}\right)^{2} v^{2} x_{5}+q^{2} v^{2}\left(x_{4} x_{5}-x_{5}^{2}\right)-4 q^{2} x_{5} x_{6}^{2}-x_{4}^{2} x_{6}^{2} .
$$

For $n_{4} \leq 0$, where we have $c_{1}^{B}(\underline{n})=c_{2}^{B}(\underline{n})=0$, the integrals in Eq. (150) can be solved analytically in the order $x_{4}, x_{5}, x_{6}$ using Eq. (39) for $x_{4}$, the formula

$$
\begin{aligned}
I_{h}^{(6)}(\alpha, \beta) & =\int_{0}^{\infty} \mathrm{d} x x^{\alpha}(x+a)^{\beta} \\
& =a^{\alpha+\beta+1} \frac{\Gamma(1+\alpha) \Gamma(-\alpha-\beta-1)}{\Gamma(-\beta)},
\end{aligned}
$$

for $x_{5}$ and Eq. (29) extended to non-integer $k_{3}$ for $x_{6}$. For $n_{4}>0$ two auxiliary master integrals, $I_{63}^{B \text {,aux }}(1,0,0,0)$ and $I_{63}^{B \text {,aux }}(1,0,1,0)$, have to be introduced where the reduction of Eq. (501) proceeds s follows

1. Reduce $n_{4}$ to one.

2. Reduce $n_{5}$ to zero.

3. The reduction of $n_{6}$ can only be performed in steps of two. Thus one end up with $n_{6}=0$ or $n_{6}=-1$.

4. Adjust the dimension, i.e., reduce $n_{d}$ to zero.

\footnotetext{
${ }^{6}$ The definition of $I_{3}^{B \text {,aux }}\left(n_{5}, n_{6}, n_{7}, n_{d}\right)$ is in analogy to Eq. (41).
} 
The corresponding recurrence relations are easily derived from Eq. (51). It is interesting to note that in Eq. (49) the master integral $I_{63}^{B \text {,aux }}(1,0,1,0)$ is canceled from $c_{1}^{B}$ and $I_{63}^{B \text {,aux }}(1,0,0,0)$ from $c_{2}^{B}$. Please note that due to the structure of Eq. (51) in addition to $I_{63}$ also a master integral with $n_{6}=-1, I_{63 a}$, has to be introduced which, however, has the same coefficient function as $I_{63}$. We want to mention that for $c_{63}$ and $c_{65}$ the master integrals $I_{6}$ and $I_{6 a}$ are needed while for $c_{64}$ and $c_{66}$ the integrals $I_{6}$ and $I_{6 a}^{B}$ are necessary.

As far as the families B2, B3 and B4 are concerned the strategy to construct the coefficient function goes along the same lines as for the families A2, A3 and A4.

For completeness let us in the following list all occurring master integrals. They have been obtained with the help of the program package developed for the calculation performed in Ref. [16] where conventional recurrence relations have been applied. In the results the overall factor $\left(i \pi^{d / 2}\right)^{2} Q^{-4 \epsilon}$ is omitted and $Q=\sqrt{-q^{2}}$ has been introduced. Moreover the standard factor $\mathrm{e}^{-2 \gamma_{\mathrm{E}} \epsilon}$ is pulled out in the formulae where an expansion in $\epsilon$ is performed. We obtain

$$
\begin{aligned}
I_{1} & =\frac{\pi}{Q^{2} v^{2}} \frac{\Gamma^{2}(5 / 2-d / 2) \Gamma^{4}(d / 2-3 / 2)}{\Gamma^{2}(d-3)} \\
I_{2} & =-\frac{\sqrt{\pi}}{Q v} \frac{\Gamma(2-d / 2) \Gamma(5 / 2-d / 2) \Gamma^{2}(d / 2-1) \Gamma^{2}(d / 2-3 / 2)}{\Gamma(d-3) \Gamma(d-2)}, \\
I_{3} & =\frac{\Gamma^{2}(2-d / 2) \Gamma^{4}(d / 2-1)}{\Gamma^{2}(d-2)}, \\
I_{4} & =-Q^{2} \frac{\Gamma(3-d) \Gamma^{3}(d / 2-1)}{\Gamma(3 d / 2-3)} \\
I_{5} & =\frac{\pi^{2}}{v^{2}}\left[-\frac{2}{3 \epsilon}-4+\left(-24+\frac{7}{9} \pi^{2}\right) \epsilon+\mathcal{O}\left(\epsilon^{2}\right)\right] \\
I_{6} & =\frac{\sqrt{\pi} Q}{v} \frac{2^{d-2} \Gamma(3-d) \Gamma(7 / 2-d) \Gamma(d / 2-1) \Gamma^{2}(d-5 / 2)}{\Gamma(2-d / 2) \Gamma(2 d-5)} \\
I_{6 a} & =-\sqrt{\pi} Q^{2} \frac{2^{d-2} \Gamma^{2}(3-d) \Gamma(d / 2-1) \Gamma^{2}(d-2)}{\Gamma(3 / 2-d / 2) \Gamma(2 d-4)} \\
I_{7} & =\frac{\sqrt{\pi} Q}{v} \frac{\Gamma(7 / 2-d) \Gamma^{2}(d / 2-1) \Gamma(d / 2-3 / 2) \Gamma(d-5 / 2)}{\Gamma(d-2) \Gamma(3 d / 2-4)} \\
I_{8} & =I_{7} \\
I_{9} & =I_{5} \\
I_{1}^{B} & =\frac{1}{2} I_{1} \\
I_{2}^{B} & =\frac{\pi^{2}}{Q v}\left[-4 \ln 2+\epsilon\left(\frac{5}{3} \pi^{2}-16 \ln 2-4 \ln ^{2} 2\right)+\mathcal{O}\left(\epsilon^{2}\right)\right] \\
I_{6 a}^{B} & =-I_{6 a} .
\end{aligned}
$$

Observe that $I_{5}=I_{9}$ and $I_{7}=I_{8}$ although the corresponding integrands are certainly different. These equalities can be immediately be seen by a simple change of variables. 
Because of $I_{7}=I_{8}$ we have in both cases one master integral less and end up with eight master integrals, $I_{1}, \ldots, I_{7}$ and $I_{6 a}$, in case A while in case $\mathrm{B}$ ten master integrals contribute, $I_{2}, \ldots, I_{7}, I_{9}, I_{6 a}^{B}, I_{1}^{B}$ and $I_{2}^{B}$. Please note that only two of them are not known in terms of $\Gamma$ functions. In the above list their result is given as an expansion in $\epsilon$ up to terms of order $\epsilon^{1}$.

A realistic diagram (in particular for arbitrary gauge parameter $\xi$ ) does not immediately lead to the integrals $F_{A}$ and $F_{B}$ defined in Eqs. (32) and (33). However, it is straightforward to map the expressions to the function (see also the appendix of Ref. [17])

$$
J(\underline{n})=\iint \frac{\mathrm{d}^{d} k \mathrm{~d}^{d} l}{\left(k^{2}\right)^{n_{1}}\left(l^{2}\right)^{n_{2}}\left[(k-q)^{2}\right]^{n_{3}}\left[(l-q)^{2}\right]^{n_{4}}\left[(k-l)^{2}\right]^{n_{5}}(v \cdot k)^{n_{6}}(v \cdot l)^{n_{7}}[v \cdot(k-l)]^{n_{8}}},
$$

with the obvious identities

$$
J\left(n_{1}, n_{2}, n_{3}, n_{4}, n_{5}, n_{6}, n_{7}, 0\right)=F_{A}(\underline{n}), J\left(n_{1}, n_{2}, n_{3}, n_{4}, n_{5}, n_{6}, 0, n_{8}\right)=F_{B}(\underline{n}) .
$$

The reduction to $F_{A}$ and $F_{B}$ is achieved with the help of the relation

$$
J(\underline{n})=J\left(n_{1}, n_{2}, n_{3}, n_{4}, n_{5}, n_{6}+1, n_{7}-1, n_{8}\right)+J\left(n_{1}, n_{2}, n_{3}, n_{4}, n_{5}, n_{6}+1, n_{7}, n_{8}-1\right),
$$

where either $n_{7}$ or $n_{8}$ are reduced to zero from positive values. In the case of negative $n_{7}$ or $n_{8}$ such a reduction is similar.

The method described above has been implemented in a Mathematica package. The typical runtime of an integral contributing to the static potential or to the $1 /\left(m_{q} r^{2}\right)$ corrections amounts to a few seconds. This extends to the order of a minute for integrals which occur in the calculation for a general gauge parameter where the exponents of the propagators can be significantly higher.

Let us at the end of this section present some explicit results which illustrate the functionality of our method. For this reason we list next to the results for $F_{A}(\underline{n})$ and $F_{B}(\underline{n})$ also the (non-zero) expressions for the individual coefficient functions.

For $\underline{n}=(2,2,1,1,1,1,1)$ we get $^{7}$

$$
F_{A}(2,2,1,1,1,1,1)=\frac{1}{\left(q^{2}\right)^{4} v^{2}}\left(\frac{2}{3 \epsilon}+\frac{4}{3 \epsilon} \pi^{2}-\frac{16}{9}+\frac{368}{45} \pi^{2}-8 \zeta(3)+\mathcal{O}(\epsilon)\right),
$$

with the coefficients

$$
\begin{aligned}
& c_{1}=\frac{2(d-5)(d-4)}{q^{6}}, \quad c_{3}=\frac{8(d-5)(d-3)^{2}}{(d-4) q^{8} v^{2}}, \\
& c_{41}=c_{42}=\frac{-3(d-3)(3 d-16)(3 d-14)(3 d-10)(3 d-8)\left(5 d^{3}-93 d^{2}+588 d-1264\right)}{(d-9)(d-8)(d-7)(d-6)^{2}(d-4)^{2} q^{10} v^{2}}, \\
& c_{51}=c_{52}=\frac{-3(3 d-17)(3 d-13)(3 d-11)}{(d-9)(d-7) q^{8}}, \\
& c_{61}=c_{62}=\frac{-32(2 d-13)(2 d-11)(2 d-9)(2 d-7)(2 d-5)}{(d-9)(d-7)(d-6)(d-4) q^{10} v^{2}},
\end{aligned}
$$

\footnotetext{
${ }^{7}$ The same standard overall factors are pulled out as in Eqs. (53).
} 
where $c_{61}$ and $c_{62}$ get multiplied by $I_{6 a}$. In the case $B$ the results read

$$
\begin{gathered}
F_{B}(2,2,1,1,1,1,1)=\frac{1}{\left(q^{2}\right)^{4} v^{2}}\left(-\frac{1}{3 \epsilon}+\frac{4}{3 \epsilon} \pi^{2}+\frac{8}{9}+\frac{368}{45} \pi^{2}+4 \zeta(3)+\mathcal{O}(\epsilon)\right), \\
c_{1}^{B}=\frac{2(d-5)(d-4)}{q^{6}}, \quad c_{3}=\frac{-4(d-5)(d-3)^{2}}{(d-4) q^{8} v^{2}}, \\
c_{41}=\frac{3(d-3)(3 d-16)(3 d-14)(3 d-10)(3 d-8)\left(7 d^{3}-117 d^{2}+654 d-1232\right)}{(d-9)(d-8)(d-7)(d-6)^{2}(d-4)^{2} q^{10} v^{2}}, \\
c_{42}=\frac{-6(d-3)(3 d-16)(3 d-14)(3 d-10)(3 d-8)\left(d^{3}-12 d^{2}+33 d+16\right)}{(d-9)(d-8)(d-7)(d-6)^{2}(d-4)^{2} q^{10} v^{2}}, \\
c_{53}=\frac{-3(3 d-17)(3 d-13)(3 d-11)}{(d-9)(d-7) q^{8}}, \\
c_{63}=c_{64}=-\frac{4(2 d-7)(2 d-5)\left(15 d^{4}-304 d^{3}+2240 d^{2}-7093 d+8118\right)}{(d-9)(d-7)(d-6)(d-4) q^{10} v^{2}}, \\
c_{65}=c_{66}=\frac{4(2 d-7)(2 d-5)\left(d^{2}-17 d+55\right)}{(d-7)(d-4) q^{10} v^{2}}, \\
c_{67}=\frac{-32(2 d-13)(2 d-11)(2 d-9)(2 d-7)(2 d-5)}{(d-9)(d-7)(d-6)(d-4) q^{10} v^{2}}, \\
c_{9}=\frac{-3(3 d-17)(3 d-13)(3 d-11)}{(d-9)(d-7) q^{8}},
\end{gathered}
$$

where $c_{63}$ and $c_{65}$ get multiplied by $I_{6 a}$ and $c_{64}, c_{66}$ and $c_{67}$ by $I_{6 a}^{B}$. As a second example let us consider $\underline{n}=(1,1,2,1,1,-1,1)$ which gives

$$
\begin{aligned}
& F_{A}(1,1,2,1,1,-1,1)=\frac{1}{\left(q^{2}\right)^{2}}\left(-\frac{1}{2 \epsilon}+\frac{3}{2}-2 \zeta(3)+\mathcal{O}(\epsilon)\right), \\
& c_{3}=\frac{2(d-3)}{(d-4) q^{4}}, \quad c_{41}=\frac{-3(3 d-10)(3 d-8)\left(d^{2}-5 d+2\right)}{2(d-6)(d-5)(d-4)^{2} q^{6}}, \\
& c_{42}=\frac{3(d-5)(d-2)(3 d-10)(3 d-8)}{2(d-6)(d-4)^{2} q^{6}} \\
& c_{62}=\frac{4(2 d-9)(2 d-7)(2 d-5)}{(d-5)(d-4) q^{6}}
\end{aligned}
$$

where $c_{62}$ gets multiplied by $I_{6 a}$.

$$
\begin{gathered}
F_{B}(1,1,2,1,1,-1,1)=\frac{1}{\left(q^{2}\right)^{2}}\left(-\frac{1}{2 \epsilon}+\frac{1}{2}+\mathcal{O}(\epsilon)\right), \\
c_{3}=\frac{(d-5)(d-3)}{(d-6) q^{4}}, \quad c_{41}=\frac{-3(3 d-10)(3 d-8)\left(d^{2}-9 d+22\right)}{2(d-6)^{2}(d-5)(d-4) q^{6}},
\end{gathered}
$$




$$
\begin{aligned}
& c_{42}=\frac{3(3 d-10)(3 d-8)\left(d^{2}-11 d+26\right)}{2(d-6)^{2}(d-4) q^{6}}, \quad c_{63}=\frac{(2 d-11)(2 d-7)(2 d-5)}{(d-6)(d-5) q^{6}} \\
& c_{64}=\frac{-(2 d-7)(2 d-5)}{(d-6)(d-5) q^{6}}, \quad c_{65}=\frac{(2 d-7)^{2}(2 d-5)}{(d-6)(d-5) q^{6}} \\
& c_{66}=\frac{-(2 d-7)(2 d-5)(4 d-19)}{(d-6)(d-5) q^{6}}
\end{aligned}
$$

where again $c_{63}$ and $c_{65}$ get multiplied by $I_{6 a}$ and $c_{64}$ and $c_{66}$ by $I_{6 a}^{B}$.

\section{Conclusion}

In this paper we described an algorithmic procedure to identify the master integrals for

a given class of Feynman diagram. Furthermore details are given for the computation of the corresponding coefficient functions which are formally defined in Eq. (7)). The general procedure was applied to useful one- and two-loop integrals which often appear in practical applications. In particular we discussed in great detail the integrals needed for the calculation of the two-loop heavy quark potential. We do hope that, with our instructions, the reader will be able to apply Eq. (7) to various recursion problems for Feynman integrals.

Let us finally point out that there is also another approach to use Eq. (7) which is based on an expansion of some parametric representations (originating from Eq. (7D) in the limit of large dimension $d$ [12. There the crucial assumption is the rational dependence of the coefficients $c_{i}$ on $d$. No description of this procedure has been published up to now.

\section{Acknowledgements}

M.S. would like to thank Y. Schröder for discussions. The work of V.S. was supported by the Russian Foundation for Basic Research through project 01-02-16171, the Volkswagen Foundation through contract No. I/77788, and INTAS through grant 00-00313.

\section{References}

[1] P.A. Baikov, Phys. Lett. B 385 (1996) 404; Nucl. Instrum. Methods A 389 (1997) 347.

[2] M. Steinhauser, Phys. Rep. 364 (2002) 247;

V.A. Smirnov, hep-ph/0209177; Nucl. Phys. Proc. Suppl. 116 (2003) 417.

[3] K.G. Chetyrkin and F.V. Tkachov, Nucl. Phys. B 192 (1981) 159.

[4] G. 't Hooft and M. Veltman, Nucl. Phys. B44 (1972) 189;

C.G. Bollini and J.J. Giambiagi, Nuovo Cim. 12B (1972) 20. 
[5] M. Beneke and V.A. Smirnov, Nucl. Phys. B522 (1998) 321;

V.A. Smirnov and E.R. Rakhmetov, Teor. Mat. Fiz. 120 (1999) 64;

V.A. Smirnov, Phys. Lett. B465 (1999) 226;

V.A. Smirnov, Applied Asymptotic Expansions in Momenta and Masses (Springer, Berlin, Heidelberg, 2001).

[6] O.V. Tarasov, Nucl. Phys. B 480 (1996) 397; Phys. Rev. D 54 (1996) 6479; Nucl. Phys. (Proc. Suppl.) 89 (2000) 237.

[7] S. Laporta, Int. J. Mod. Phys. A 15 (2000) 5087.

[8] A.V. Kotikov, Phys. Lett. B254 (1991) 158; B259 (1991) 314; B267 (1991) 123.

[9] T. Gehrmann and E. Remiddi, Nucl. Phys. B 580 (2000) 485; Nucl. Phys. B 601 (2001) 248; Nucl. Phys. B 601 (2001) 287.

[10] P.A. Baikov and V.A. Smirnov, Phys. Lett. B 477 (2000) 367.

[11] P.A. Baikov and M. Steinhauser, Comput. Phys. Commun. 115 (1998) 161.

[12] P.A. Baikov, K.G. Chetyrkin, and J.H. Kühn, Phys. Rev. Lett. 88 (2002) 012001; Phys. Rev. D67 (2003) 074026; Phys. Lett. B559 (2003) 245.

[13] M. Peter, Phys. Rev. Lett. 78, 602 (1997); Nucl. Phys. B501, 471 (1997).

[14] Y. Schröder, Phys. Lett. B 447, 321 (1999).

[15] Y. Schröder, Ph.D. thesis (Hamburg, 1999), DESY-THESIS-1999-021.

[16] B.A. Kniehl, A.A. Penin, V.A. Smirnov, and M. Steinhauser, Phys. Rev. D65 (2002) 091503.

[17] B. A. Kniehl, A. A. Penin, V. A. Smirnov, and M. Steinhauser, Nucl. Phys. B 635 (2002) 357. 